\title{
Modeling Power Saving for GAN and UMTS Interworking
}

\author{
Shun-Ren Yang, Member, IEEE, Phone Lin, Senior Member, IEEE, and Pei-Tang Huang
}

\begin{abstract}
GPP 43.318 specifies the Generic Access Network (GAN) for interworking between Wireless Local Area Network (WLAN) and Universal Mobile Telecommunications System (UMTS) core network. A dual-mode Mobile Station (MS) is equipped with two communication modules to support both WLAN and UMTS radio technologies, which shortens the battery lifetime of the MS. This paper proposes an analytical model and conducts simulation experiments to study the power consumption of dual-mode MSs in terms of the power consumption indicator and mean packet waiting time. Our study provides guidelines for designing WLAN-UMTS dual-mode MSs.
\end{abstract}

Index Terms-Generic access network (GAN), power saving, universal mobile telecommunications system (UMTS), wireless local area network (WLAN).

\section{INTRODUCTION}

I EEE 802.11 Wireless Local Area Network (WLAN) provides users high bit-rate wireless transmission service within hot-spot areas, e.g., indoor or basement. On the other hand, 3GPP Universal Mobile Telecommunications System (UMTS) provides wireless transmission service within wide areas and supports high user mobility. WLAN and UMTS are treated as complementary wireless network technologies [6]. To provide users wireless access service to networks irrespective of their locations and network access technologies, the Unlicensed Mobile Access (UMA) technology [18] is proposed for interworking and integration between UMTS and WLAN, which has been proven and accommodated in 3GPP 43.318 [1]. The Generic Access Network (GAN) is defined in 3GPP 43.318 to enable WLAN to connect to the UMTS core network.

Manuscript received August 23, 2007; revised January 4, 2008; accepted February 15, 2008. The associate editor coordinating the review of this paper and approving it for publication was W. Lou.

The work of S.-R. Yang was supported in part by the National Science Council (NSC) of Taiwan under Contracts NSC-95-2752-E-007-003-PAE, NSC-95-2221-E-007-026 and NSC-95-2219-E-007-011, and the Institute for Information Industry (III). P. Lin's work was sponsored in part by the National Science Council (NSC), R.O.C., under the contract number NSC-96-2627-E002-001, NSC-96-2811-E-002-010, NSC-96-2628-E-002-002-MY2 and NSC95-2221-E-002-091-MY3, Ministry of Economic Affairs (MOEA), R.O.C., under contract number 93-EC-17-A-05-S1-0017, Telcordia Applied Research Center, Taiwan Network Information Center (TWNIC), and Excellent Research Projects of National Taiwan University, 97R0062-05.

S.-R. Yang is with the Department of Computer Science and Institute of Communications Engineering, National Tsing Hua University, Hsinchu, Taiwan 300, R.O.C. (e-mail: sryang@cs.nthu.edu.tw).

P. Lin is with the Department of Computer Science \& Information Engineering and Graduate Institute of Networking \& Multimedia, National Taiwan University, Taipei 106, Taiwan, R.O.C. (e-mail: plin@csie.ntu.edu.tw).

P.-T. Huang is with the Telecommunication Laboratories, Chunghwa Telecom Company, Ltd., Taipei, Taiwan 10682, R.O.C. (e-mail: beta@cht.com.tw).

Digital Object Identifier 10.1109/T-WC.2008.070936
GAN provides UMTS subscribers with the low-cost and high-speed WLAN access. However, a dual-mode Mobile Station (MS) is equipped with two communication modules for both WLAN and UMTS radio technologies. The work in [17] showed that the battery lifetime will be significantly shortened while an additional WLAN module is added to an MS. Therefore, how to reduce dual-mode MS power consumption is an important issue, which may reflect the user satisfaction with the offered wireless access service. Many research efforts in the literature have dedicated to the investigation of the MS power saving mechanisms in different wireless mobile networks, e.g., [14] for CDPD, [19] for UMTS, [12] for both UMTS and cdma2000, and [20] for IEEE 802.11 WLAN. Nevertheless, all of these studies only considered the power consumption behavior of single-mode MSs. To the best of our knowledge, there is no previous work covering the "power saving of a dual-mode MS" topic.

This paper proposes an analytical model to study the power consumption issue for MSs operating in the GAN and UMTS interworking network. The model quantifies the power consumption of a WLAN-UMTS dual-mode MS, which is referred to as the power consumption indicator. It is clear that the lower the power consumption indicator, the more effective the utilized power management technique. However, reducing the power consumption may at the same time degrade the system performance in terms of service delay. Therefore, we also quantify the mean packet waiting time to examine the penalty caused by exercising the power saving mechanism for GAN-UMTS interworking. Due to the complicated behavior of a dual-mode MS, the proposed analytical model may not well capture the MS behavior under some conditions. To release these constraints of the analytical model, we conduct simulation experiments as well. We note that the performance of a power saving mechanism primarily depends on MSs' uplink and downlink packet transmission/reception behavior. Whenever an MS has uplink packets to transmit, it can immediately terminate the power saving operation and switch into the power active state for packet delivery. On the other hand, for the downlink packet reception, it is very difficult for MSs to predict the instants of the subsequent packet arrivals. In this case, an MS can not adjust its power management state proactively to adapt to the downlink packet traffic. This paper will concentrate on the more challenging power management for MS downlink packet transmissions. The uplink performance metrics such as the transmission power consumption of MSs are therefore not discussed in this paper. Our work derives close-form equations for both the power consumption indicator and mean packet waiting time with the premise that 


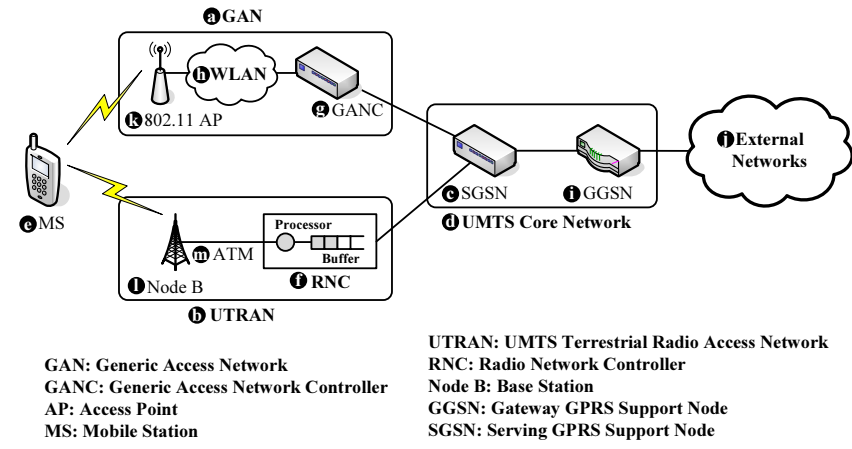

Fig. 1. The system model for the GAN and UMTS interworking

the WLAN available and unavailable periods are sufficiently small. Furthermore, our study indicates that with proper parameter settings, power management techniques can reduce the power consumption indicator of a WLAN-UMTS dual-mode MS without significantly increasing the mean packet waiting time. The analytical and simulation results of this work can serve as guidelines for the implementation of WLAN-UMTS dual-mode MSs.

\section{SySTEM ModeL}

This section first gives an overview of the GAN-UMTS interworking architecture, and then describes the system model for our study of GAN-UMTS dual-mode MS power saving.

Figure 1 illustrates a simplified system architecture for the GAN-UMTS interworking, where GAN (Figure 1 (a)) is an alternative radio access network for the UMTS core network (Figure 1 (d)). We may apply any kind of IP access technologies in GAN, such as IEEE 802.11 [9] or Bluetooth [3]. This paper assumes IEEE 802.11 WLAN (Figure 1 (h)) as the underlying IP access technology in GAN. Both GAN and UMTS Terrestrial Radio Access Network (UTRAN; Figure 1 (b)) connect to Serving GPRS Support Node (SGSN; Figure 1 (c)) in the UMTS core network and receive packets destined to a dual-mode MS (Figure 1 (e)) from external IP networks (Figure 1 (j)). Functioning like Radio Network Controller (RNC; Figure 1 (f)) in UTRAN, Generic Access Network Controller (GANC; Figure $1(\mathrm{~g})$ ) in GAN receives packets from SGSN and forwards them to the MS through Access Points (APs; Figure $1(\mathrm{k})$ ) in WLAN. While the MS leaves WLAN coverage, the SGSN may also forward the incoming packets to the RNC in the UTRAN. The RNC processor sends the packets to the Node B (Figure 1 (l)) through an Asynchronous Transfer Mode (ATM; Figure 1 (m)) link. The Node B then delivers the packets to the MS through the Wideband Code Division Multiple Access (WCDMA) radio link.

To conserve the power budget of a GAN-UMTS dual-mode MS, the UMTS Discontinuous Reception (DRX) [2] and the IEEE 802.11 Power Saving Mode (PSM) [9] are employed, respectively. The concept of DRX is for an idle MS to power off the radio receiver for a predefined period (referred as $D R X$ cycle) instead of continuously listening to the radio channel signal. As shown in Figure 2, the activities of an MS's UMTS receiver module under DRX can be characterized in terms of three periods:

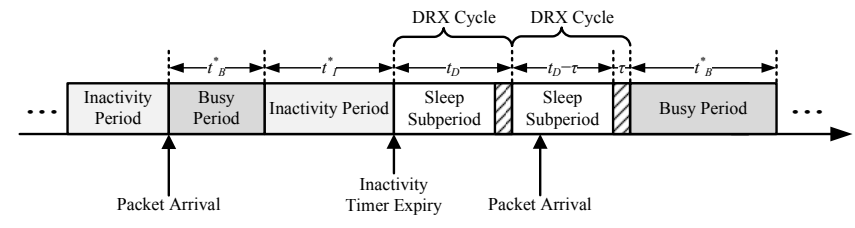

Fig. 2. UMTS Discontinuous Reception (DRX) mechanism

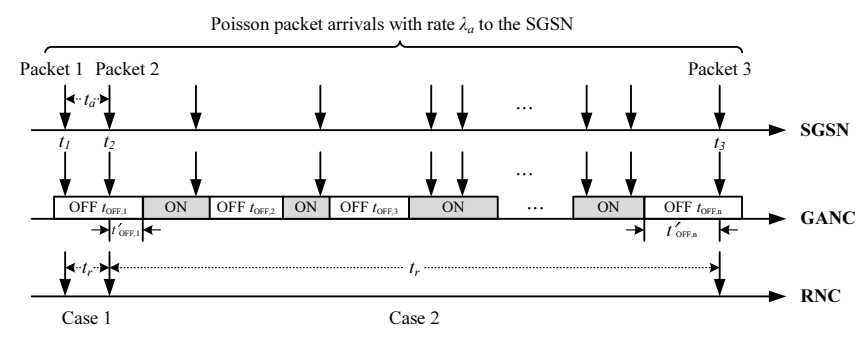

Fig. 3. Timing diagram

Busy periods. During packet transmission to the MS, incoming packets are first stored in the RNC buffer before they are delivered to the MS. Then, the RNC processor transmits the packets in the First In First Out (FIFO) order.

Inactivity periods. When the RNC buffer becomes empty, the RNC inactivity timer is activated. If any packet arrives at the RNC before the inactivity timer expires, the timer is stopped. The RNC processor starts to transmit packets, and another busy period begins.

Sleep periods. If no packet arrives before the inactivity timer expires, the MS enters a sleep period, and the UMTS receiver module is turned off. The sleep period contains one or more DRX cycles. At the end of a DRX cycle, the MS wakes up to listen to the paging channel. If some packets have arrived at the RNC during the last DRX cycle, the MS starts to receive packets and the sleep period ends. Otherwise, the MS returns to sleep until the end of the next DRX cycle.

Note that during busy and inactivity periods, the MS turns on the UMTS receiver module.

Zheng et al. [20] have shown that the IEEE 802.11 PSM mechanism is oblivious of the packet traffic characteristics, and thus is not energy-efficient under light traffic load and suffer from significant performance degradation at higher traffic load in terms of power consumption and packet mean waiting time. Therefore, our system model considers UMTS DRX mechanism during packet transmission through UMTS but ignores IEEE 802.11 PSM during packet transmission through IEEE 802.11 WLAN.

As shown in Figure 3, since the WLAN can support higher data transmission rate and is of lower cost, we suppose that the SGSN delivers the packets to the GANC whenever possible. The SGSN could also utilize the global always-on UMTS connectivity for packet delivery when the MS leaves the WLAN hotspot coverage and the WLAN connection is not available. Suppose that packet arrivals for an MS to the SGSN form a Poisson stream with rate $\lambda_{a}$. We assume that the WLAN availability for the MS follows the ON-OFF patterns repeatedly. Specifically, the WLAN connectivity is available 
during an ON period, and is unavailable when the ON period ends. Then, the WLAN connectivity enters an OFF period. The ON and OFF periods are assumed to be exponentially distributed with rates $\lambda_{o}$ and $\lambda_{f}$, respectively.

Due to the high wireless transmission rate feature of WLAN, we assume that each packet arrival to the GANC can be transmitted immediately, and no packet has to be buffered in the GANC. When packets arrive during a WLAN OFF period, they are forwarded to the RNC. In UTRAN, ATM is much faster and more reliable than the WCDMA wireless transmission. Therefore, we ignore the ATM transmission delay between the RNC and the Node B, and the RNC and the Node B are modeled as a FIFO queueing server. Let $t_{x}$ denote the packet service time, i.e., the interval between the time when a packet is transmitted by the RNC processor and the time when the corresponding ack is received by the RNC processor. Let $t_{I}$ be the threshold of the RNC inactivity timer, and $t_{D}$ be the length of the UMTS DRX cycle. At the end of every DRX cycle, the MS must wake up for a short period $\tau$ so that it can listen to the paging information from the network. Therefore the "power saving" period in a DRX cycle is $t_{D}-\tau$.

\section{An AnALYTIC Model}

This section proposes an analytical model to investigate the power consumption of a dual-mode MS. In the following, we first determine the packet arrival process to the RNC. Then, based on the inter-packet arrival time distribution to the RNC, we derive the following two output measures:

- the power consumption indicator $P_{i}$ : the average power consumption of an MS's radio receivers (including the UMTS and the WLAN receiver modules) when the UMTS DRX mechanism is exercised;

- the mean packet waiting time $E\left[t_{w}\right]$ : the expected waiting time for a packet between the time when it is received by the SGSN and the time when it is transmitted by the GANC or the RNC to the MS.

As shown in Figure 3, two cases are considered to derive the probability density function $f_{r}(t)$ for the inter-packet arrival time $t_{r}$ between two consecutive packet arrivals to the RNC.

Case 1In this case, two consecutive packets, Packets 1 and 2 , arrive at the SGSN within the same WLAN OFF period denoted as $t_{\mathrm{OFF}, 1}$, and both Packets 1 and 2 are forwarded to the RNC. Let $t_{1}$ and $t_{2}$ be the arrival time points of Packets 1 and 2, respectively. The interval $t_{2}-t_{1}$ has the following probability density function

$$
f_{t_{2}-t_{1}}(t)=f_{a}(t) \operatorname{Pr}\left[t_{\mathrm{OFF}, 1}>t\right],
$$

where $f_{a}(t)$ is the probability density function of the inter-packet arrival time $t_{a}$ to the SGSN, and $\operatorname{Pr}\left[t_{\mathrm{OFF}, 1}>t\right]$ is the probability that two consecutive packets arrive during $t_{\mathrm{OFF}, 1}$. Since $t_{a}$ and $t_{\mathrm{OFF}, 1}$ are assumed to be exponentially distributed with rate $\lambda_{a}$ and $\lambda_{f}$, (1) can be rewritten as

$$
f_{t_{2}-t_{1}}(t)=e^{-\lambda_{f} t} \lambda_{a} e^{-\lambda_{a} t} .
$$

In this case, the inter-packet arrival time $t_{r}$ to the RNC equals to $t_{2}-t_{1}$. Thus, the probability density function $f_{r}(t)$ for $t_{r}$ is

$$
f_{r}(t)=f_{t_{2}-t_{1}}(t)=e^{-\lambda_{f} t} \lambda_{a} e^{-\lambda_{a} t} .
$$

Case 2After the arrival of Packet 2, let Packet 3 arrival at $t_{3}$ be the first packet arrival during a WLAN OFF period. In this case, Packets 2 and 3 are separated by several packet arrivals within WLAN ON periods. The inter-packet arrival time $t_{r}$ for Case 2 is equal to $t_{3}-t_{2}$. Let $t_{f}$ be the total length of WLAN OFF periods during $\left[t_{2}, t_{3}\right]$. Assume that there are $n$ WLAN OFF periods $t_{\mathrm{OFF}, 1}, t_{\mathrm{OFF}, 2}, \ldots, t_{\mathrm{OFF}, n}$ between $t_{2}$ and $t_{3}$. Denote $t_{\mathrm{OFF}, 1}^{\prime}$ as the residual OFF period of $t_{\mathrm{OFF}, 1}$ at $t_{2}$ and $t_{\mathrm{OFF}, n}^{\prime}$ as the age $\mathrm{OFF}$ period of $t_{\mathrm{OFF}, n}$ at $t_{3}$. Then,

$$
t_{f}=t_{\mathrm{OFF}, 1}^{\prime}+\left(\sum_{i=2}^{n-1} t_{\mathrm{OFF}, i}\right)+t_{\mathrm{OFF}, n}^{\prime} .
$$

As shown in Figure 3, no packet arrives during $t_{\mathrm{OFF}, 1}^{\prime}$ (with probability $e^{-\lambda_{a} t_{\mathrm{OFF}, 1}^{\prime}}$ ), $t_{\mathrm{OFF}, 2}$ (with probability $\left.e^{-\lambda_{a} t} \mathrm{OFF}, 2\right), \ldots$, and $t_{\mathrm{OFF}, n-1}$ (with probability $e^{-\lambda_{a} t} \mathrm{OFF}, n-1_{1}$ ), and Packet 3 arrives at time $t_{\mathrm{OFF}, n}^{\prime}$ of the WLAN OFF period $t_{\mathrm{OFF}, n}$ (with probability density function $\lambda_{a} e^{-\lambda_{a} t_{\mathrm{OFF}, n}^{\prime}}$. Therefore, the probability density function $f_{f}\left(t_{f}\right)$ for $t_{f}$ is expressed as equation 5 .

Let $N_{o}$ be the number of WLAN ON periods during $\left[t_{2}, t_{3}\right]$, and $t_{o}$ be the total length of these $N_{o}$ WLAN ON periods. We proceed to derive the conditional probability mass function $\theta_{n \mid t_{f}}=\operatorname{Pr}\left[N_{o}=n \mid t_{f}\right]$ for $N_{o}$ and the conditional probability density function $f_{o \mid t_{f}, N_{o}}\left(t_{o} \mid t_{f}, N_{o}=n\right)$ for $t_{o}$. In Figure $3, t_{\mathrm{OFF}, 1}^{\prime}$, $t_{\mathrm{OFF}, i}(2 \leq i \leq n-1)$, and $t_{\mathrm{OFF}, n}^{\prime}$ are all exponential random variables with rate $\lambda_{f}$. Therefore, it is clear that the number of WLAN ON periods has the Poisson distribution with rate $\lambda_{f}$, and we have

$$
\theta_{n \mid t_{f}}=\operatorname{Pr}\left[N_{o}=n \mid t_{f}\right]=e^{-\lambda_{f} t_{f}} \frac{\left(\lambda_{f} t_{f}\right)^{n}}{n !} .
$$

Conditioning on $t_{f}$ (with probability $f_{f}\left(t_{f}\right) d t_{f}$ ) and $N_{o}=n$ (with probability $\theta_{n \mid t_{f}}$ ), the total length $t_{o}$ of WLAN ON periods during $\left[t_{2}, t_{3}\right]$ is an Erlang- $n$ random variable with rate $\lambda_{o}$ and probability density function

$$
f_{o \mid t_{f}, N_{o}}\left(t_{o} \mid t_{f}, N_{o}=n\right)=\frac{\lambda_{o}^{n}}{(n-1) !} t_{o}^{n-1} e^{-\lambda_{o} t_{o}} .
$$

The $f_{r}(t)$ for the inter-packet arrival time $t_{r}$ in this case can then be expressed in terms of $f_{f}\left(t_{f}\right), \theta_{n \mid t_{f}}$ and $f_{o \mid t_{f}, N_{o}}\left(t_{o} \mid t_{f}, N_{o}=n\right)$ as equation 8. Substitute (5), (6), and (7) into (8) to obtain

$$
\begin{aligned}
f_{r}(t)=\int_{t_{f}=0}^{t} & \lambda_{a} e^{-\lambda_{a} t_{f}} \\
\times & \left\{\sum_{n=1}^{\infty} e^{-\lambda_{f} t_{f}}\left[\frac{\left(\lambda_{f} t_{f}\right)^{n}}{n !}\right]\left[\frac{\lambda_{o}^{n}}{(n-1) !}\right]\right. \\
& \left.\times\left(t-t_{f}\right)^{n-1} e^{-\lambda_{o}\left(t-t_{f}\right)} d t_{f}\right\} .
\end{aligned}
$$




$$
\begin{aligned}
f_{f}\left(t_{f}\right) & =e^{-\lambda_{a} t_{\mathrm{OFF}, 1}^{\prime}} e^{-\lambda_{a} t_{\mathrm{OFF}, 2} \cdots e^{-\lambda_{a} t_{\mathrm{OFF}, n-1}} \lambda_{a} e^{-\lambda_{a} t_{\mathrm{OFF}, n}^{\prime}}} \\
& =\lambda_{a} e^{-\lambda_{a}\left(t_{\mathrm{OFF}, 1}^{\prime}+t_{\mathrm{OFF}, 2}+\cdots+t_{\mathrm{OFF}, n-1}+t_{\mathrm{OFF}, n}^{\prime}\right)} \\
& =\lambda_{a} e^{-\lambda_{a} t_{f}} \\
f_{r}(t) & =\int_{t_{f}=0}^{t} f_{f}\left(t_{f}\right) \sum_{n=1}^{\infty} \theta_{n \mid t_{f}} f_{o \mid t_{f}, N_{o}}\left(t-t_{f} \mid t_{f}, N_{o}=n\right) d t_{f} .
\end{aligned}
$$

Combining (3) and (9), we derive the probability density function $f_{r}(t)$ for the inter-packet arrival time $t_{r}$ to the RNC

$$
\begin{aligned}
f_{r}(t)=e^{-\lambda_{f} t} \lambda_{a} e^{-\lambda_{a} t} & \\
+ & \int_{t_{f}=0}^{t} \lambda_{a} e^{-\lambda_{a} t_{f}} \\
\quad & \left\{\sum_{n=1}^{\infty} e^{-\lambda_{f} t_{f}}\left[\frac{\left(\lambda_{f} t_{f}\right)^{n}}{n !}\right]\left[\frac{\lambda_{o}^{n}}{(n-1) !}\right]\right. \\
& \left.\quad \times\left(t-t_{f}\right)^{n-1} e^{-\lambda_{o}\left(t-t_{f}\right)} d t_{f}\right\} .
\end{aligned}
$$

Equation (10) is too complicated for the analysis of $P_{i}$ and $E\left[t_{w}\right]$. With the following theorem, we attempt to obtain a simpler $f_{r}(t)$ probability density function.

Theorem 1: When $\lambda_{o} \rightarrow \infty, \lambda_{f} \rightarrow \infty$ and $\frac{\lambda_{f}}{\lambda_{o}} \rightarrow C>0$ where $C$ is a constant, we have that the inter-packet arrival time $t_{r}$ to the RNC follows an exponential distribution with rate $\frac{\lambda_{a}}{1+C}$.

Proof. Since $N_{o} \mid t_{f}$ is a Poisson random variable with rate $\lambda_{f}$ (see (6)), we have

$$
E\left[N_{o} \mid t_{f}\right]=\lambda_{f} t_{f} \text { and } \operatorname{Var}\left[N_{o} \mid t_{f}\right]=\lambda_{f} t_{f} .
$$

Furthermore, $t_{o} \mid t_{f}, N_{o}=n$ is an Erlang- $n$ random variable with rate $\lambda_{o}$ (see (7)), and therefore

$$
E\left[t_{o} \mid t_{f}, N_{o}=n\right]=\frac{n}{\lambda_{o}} \text { and } \operatorname{Var}\left[t_{o} \mid t_{f}, N_{o}=n\right]=\frac{n}{\lambda_{o}^{2}} .
$$

Using expectation by conditioning technique [16], we have that

$$
E\left[t_{o} \mid t_{f}\right]=E\left[E\left[t_{o} \mid t_{f}, N_{o}\right] \mid t_{f}\right] .
$$

From (11) and (12), (13) is rewritten as

$$
E\left[t_{o} \mid t_{f}\right]=E\left[\frac{N_{o}}{\lambda_{o}} \mid t_{f}\right]=\frac{\lambda_{f} t_{f}}{\lambda_{o}} .
$$

According to [16, page 51], the variance $\operatorname{Var}\left[t_{o} \mid t_{f}\right]$ can be expressed as

$$
\begin{aligned}
\operatorname{Var}\left[t_{o} \mid t_{f}\right]= & \operatorname{Var}\left[E\left[t_{o} \mid t_{f}, N_{o}\right] \mid t_{f}\right] \\
& +E\left[\operatorname{Var}\left[t_{o} \mid t_{f}, N_{o}\right] \mid t_{f}\right] .
\end{aligned}
$$

Substitute (11) and (12) into (15) to yield

$$
\begin{aligned}
\operatorname{Var}\left[t_{o} \mid t_{f}\right] & =\operatorname{Var}\left[\frac{N_{o}}{\lambda_{o}} \mid t_{f}\right]+E\left[\frac{N_{o}}{\lambda_{o}^{2}} \mid t_{f}\right] \\
& =\frac{\lambda_{f} t_{f}}{\lambda_{o}^{2}}+\frac{\lambda_{f} t_{f}}{\lambda_{o}^{2}}=\frac{2 \lambda_{f} t_{f}}{\lambda_{o}^{2}} .
\end{aligned}
$$

From (14) and (16), it is clear that when $\lambda_{o} \rightarrow \infty, \lambda_{f} \rightarrow \infty$ and $\frac{\lambda_{f}}{\lambda_{o}} \rightarrow C, E\left[t_{o} \mid t_{f}\right] \rightarrow C t_{f}$ and $\operatorname{Var}\left[t_{o} \mid t_{f}\right] \rightarrow 0$, that is, $t_{o} \mid t_{f}$ converges to a constant $C t_{f}$. In this case, we have

$$
\begin{aligned}
\operatorname{Pr}\left[t_{r} \leq t\right] & =\operatorname{Pr}\left[t_{o}+t_{f} \leq t\right] \\
& \rightarrow \operatorname{Pr}\left[t_{f}+C t_{f} \leq t\right] \\
& =\operatorname{Pr}\left[t_{f} \leq \frac{t}{1+C}\right]=1-e^{-\left(\frac{\lambda_{a}}{1+C}\right) t} .
\end{aligned}
$$

Namely, $t_{r}$ has the exponential distribution with rate $\frac{\lambda_{a}}{1+C}$.

Based on Theorem 1, we suppose that the inter-packet arrival time $t_{r}$ to the RNC is exponentially distributed with rate $\lambda_{r}=\frac{\lambda_{a}}{1+C}$, and we can obtain close-form equations for $P_{i}$ and $E\left[t_{w}\right]$.

The activities of an MS's UMTS receiver module can be characterized by a regenerative process [16], where a regeneration cycle consists of an inactivity period $t_{I}^{*}$, a sleep period $t_{S}^{*}$ and a busy period $t_{B}^{*}$ [19]. Let $P_{u, b}, P_{u, i}, P_{u, s}$, and $P_{u, l}$ be the power consumption (in watts) of the UMTS receiver module in the UMTS busy period, inactivity period, sleep period, and listening period (at the end of each DRX cycle), respectively. Let $P_{w, o}$ and $P_{w, f}$ be the power consumption (in watts) of the WLAN receiver module when the WLAN connection is available and unavailable, respectively. Suppose that there are $N$ DRX cycles in a sleep period. Based on [16, Theorem 3.7.1], the average power consumption $P_{i, u}$ of the UMTS receiver module can be expressed as equation 17. Suppose that the $t_{x}$ distribution has mean $1 / \lambda_{x}$ and variance $V_{x}$. Let $t_{I}=1 / \lambda_{I}$ and $t_{D}=1 / \lambda_{D} . E\left[t_{I}^{*}\right], E\left[t_{S}^{*}\right], E[N]$, and $E\left[t_{B}^{*}\right]$ have been derived in [19], which are expressed as

$$
\begin{aligned}
& E\left[t_{I}^{*}\right]=\left(\frac{1}{\lambda_{r}}\right)\left(1-e^{-\lambda_{r} / \lambda_{I}}\right), \\
& E\left[t_{S}^{*}\right]=\frac{e^{-\lambda_{r} / \lambda_{I}}}{\left(1-e^{-\lambda_{r} / \lambda_{D}}\right) \lambda_{D}}, \\
& E[N]=\frac{e^{-\lambda_{r} / \lambda_{I}}}{1-e^{-\lambda_{r} / \lambda_{D}}}, \text { and } \\
& E\left[t_{B}^{*}\right]=\frac{\rho\left[\lambda_{D}\left(1-e^{-\lambda_{r} / \lambda_{I}}\right)\left(1-e^{-\lambda_{r} / \lambda_{D}}\right)+\lambda_{r} e^{-\lambda_{r} / \lambda_{I}}\right]}{\lambda_{r} \lambda_{D}\left(1-e^{-\lambda_{r} / \lambda_{D}}\right)(1-\rho)},
\end{aligned}
$$

where $\rho=\lambda_{r} / \lambda_{x}$. Substituting (18) into (17), we could obtain $P_{i, u}$. Similarly, the activities of an MS's WLAN receiver module can be characterized by an alternating $O N-O F F$ process [16]. Therefore, the average power consumption $P_{i, w}$ of the WLAN receiver module is

$$
P_{i, w}=\frac{\lambda_{f} P_{w, o}+\lambda_{o} P_{w, f}}{\lambda_{o}+\lambda_{f}} .
$$

Combine (17) and (19) to have the power consumption indicator $P_{i}=P_{i, u}+P_{i, w}$. 


$$
\begin{aligned}
P_{i, u}=\frac{E\left[t_{I}^{*}\right] P_{u, i}+\left(E\left[t_{S}^{*}\right]-E[N] \tau\right) P_{u, s}+E[N] \tau P_{u, l}+E\left[t_{B}^{*}\right] P_{u, b}}{E\left[t_{I}^{*}\right]+E\left[t_{S}^{*}\right]+E\left[t_{B}^{*}\right]} . \\
E\left[t_{w, u}\right]=\frac{\lambda_{r} e^{-\lambda_{r} / \lambda_{I}}}{2\left[\lambda_{D}^{2}\left(1-e^{-\lambda_{r} / \lambda_{I}}\right)\left(1-e^{-\lambda_{r} / \lambda_{D}}\right)+\lambda_{r} \lambda_{D} e^{-\lambda_{r} / \lambda_{I}}\right]} \\
+\frac{\lambda_{r}\left(1+V_{x} \lambda_{x}^{2}\right)}{2(1-\rho) \lambda_{x}^{2}} .
\end{aligned}
$$

The UMTS DRX can be modeled as a variant of the $M / G / 1$ queue with multiple vacations [19], and we have derived the mean packet waiting time $E\left[t_{w, u}\right]$ in the RNC buffer in [19]. The derivation of $E\left[t_{w, u}\right]$ is summarized as follows. By constructing an embedded Markov chain for the queue length of the RNC buffer, we can first obtain the probability generating function for the steady-state queue length distribution. Then, with this probability generating function and based on [5, Theorem 4.2], we can further derive the Laplace transform for the packet waiting time $t_{w, u}$. (Let $\tilde{x}$ denote a random period of time, and let $F_{\tilde{x}}^{*}(s)$ denote the Laplace transform of $\tilde{x}$. Let $\tilde{y}$ denote the number of events from a Poisson process with rate $\lambda$ that occur during the period of time $\tilde{x}$. [5, Theorem 4.2] states that the probability generating function $\mathcal{F}_{\tilde{y}}(z)$ for $\tilde{y}$ is given by $F_{\tilde{x}}^{*}(\lambda[1-z])$.) Finally, the mean packet waiting time $E\left[t_{w, u}\right]$ of $t_{w, u}$ can be obtained from its Laplace transform by using differentiation, and is expressed as equation 20 .

Note that a packet will be served by the RNC only if it arrives at the SGSN during a WLAN OFF period (with probability $\frac{\lambda_{o}}{\lambda_{o}+\lambda_{f}}$ ). Therefore, the mean packet waiting time $E\left[t_{w}\right]$ is

$$
E\left[t_{w}\right]=\left(\frac{\lambda_{o}}{\lambda_{o}+\lambda_{f}}\right) E\left[t_{w, u}\right] .
$$

Substituting (20) into (21), we have the close-form equation for $E\left[t_{w}\right]$.

The analytical model are validated against the simulation experiments. The simulation model follows an event-driven approach which has been widely adopted in many mobile networking studies [11], [13]. The details of the simulation model are described in Appendix A. In the simulation model, we consider a more general case, i.e., release the assumptions in Theorem 1. Table I compares the analysis and simulation results for $P_{i}$ and $E\left[t_{w}\right]$, where following Theorem 1, we set $V_{x}=1 / \lambda_{x}^{2}, t_{I}=60 / \lambda_{x}, t_{D}=10 / \lambda_{x}, \tau=0.1 / \lambda_{x}$, and $\lambda_{a}=0.1 \lambda_{x}$, and following the statistics data in [4], we set $P_{u, b}=1.8, P_{u, i}=0.4, P_{u, s}=0.004, P_{u, l}=0.5, P_{w, o}=0.6$, and $P_{w, f}=0.002$ (all in watts). The table indicates that the error rate (discrepancy) between analysis and simulation is within $0.41 \%$ in all cases. It is clear that the analysis is consistent with the simulation results. Table I also shows that $\lambda_{o} \geq 10^{1} \lambda_{x}$ is sufficient for ensuring the applicability of Theorem 1 .

\section{Performance Evaluation}

This section investigates the $P_{i}$ and $E\left[t_{w}\right]$ performance for a dual-mode MS based on our simulation experiments. We adopt the ETSI traffic model [7] as the packet traffic model
TABLE I

COMPARISON BETWEEN THE ANALYSIS AND SIMULATION RESULTS $\left(V_{x}=1 / \lambda_{x}^{2}, t_{I}=60 / \lambda_{x}, t_{D}=10 / \lambda_{x}, \tau=0.1 / \lambda_{x}\right.$, AND $\left.\lambda_{a}=0.1 \lambda_{x}\right)$

\begin{tabular}{l|c|c|c|c|c}
\hline \multicolumn{7}{c}{ Power consumption indicator $\left(\lambda_{o}=10 \lambda_{x}\right)$} \\
\hline$\lambda_{f}$ & $10^{-2} \lambda_{o}$ & $10^{-1} \lambda_{o}$ & $10^{0} \lambda_{o}$ & $10^{1} \lambda_{o}$ & $10^{2} \lambda_{o}$ \\
\hline$P_{i}$ (Analytical) & 0.545077 & 0.581327 & 0.747810 & 0.729542 & 0.627239 \\
\hline$P_{i}$ (Simulation) & 0.545242 & 0.581309 & 0.747863 & 0.729607 & 0.627323 \\
\hline Error & $0.03034 \%$ & $0.00302 \%$ & $0.00718 \%$ & $0.00886 \%$ & $0.01335 \%$ \\
\hline
\end{tabular}

\begin{tabular}{l|c|c|c|c|c}
\hline \multicolumn{7}{|c}{ Mean packet waiting time $\left(\lambda_{o}=10 \lambda_{x}\right)$} \\
\hline$\lambda_{f}$ & $10^{-2} \lambda_{o}$ & $10^{-1} \lambda_{o}$ & $10^{0} \lambda_{o}$ & $10^{1} \lambda_{o}$ & $10^{2} \lambda_{o}$ \\
\hline$E\left[t_{w}\right]$ (Analytical) & 0.129287 & 0.120440 & 0.182379 & 0.269256 & 0.046673 \\
\hline$E\left[t_{w}\right]$ (Simulation) & 0.129425 & 0.120748 & 0.182674 & 0.269372 & 0.046861 \\
\hline Error & $0.10719 \%$ & $0.25549 \%$ & $0.16206 \%$ & $0.04283 \%$ & $0.40427 \%$ \\
\hline
\end{tabular}

\begin{tabular}{l|c|c|c|c|c}
\hline \multicolumn{7}{c}{ Approximation for Theorem $1\left(\lambda_{f}=10^{0} \lambda_{o}\right)$} \\
\hline$\lambda_{o}$ & $10^{-5} \lambda_{x}$ & $10^{-3} \lambda_{x}$ & $10^{-1} \lambda_{x}$ & $10^{0} \lambda_{x}$ & $10^{1} \lambda_{x}$ \\
\hline$P_{i}$ (Analytical) & 0.747810 & 0.747810 & 0.747810 & 0.747810 & 0.747810 \\
\hline$P_{i}$ (Simulation) & 0.574693 & 0.581841 & 0.728581 & 0.746172 & 0.747612 \\
\hline Error & $23.1499 \%$ & $22.1940 \%$ & $2.57138 \%$ & $0.21904 \%$ & $0.02648 \%$ \\
\hline$E\left[t_{w}\right]$ (Analytical) & 0.182379 & 0.182379 & 0.182379 & 0.182379 & 0.182379 \\
\hline$E\left[t_{w}\right]$ (Simulation) & 0.066068 & 0.099804 & 0.303903 & 0.202309 & 0.185555 \\
\hline Error & $63.7743 \%$ & $45.2766 \%$ & $66.6327 \%$ & $10.9278 \%$ & $1.74143 \%$ \\
\hline
\end{tabular}

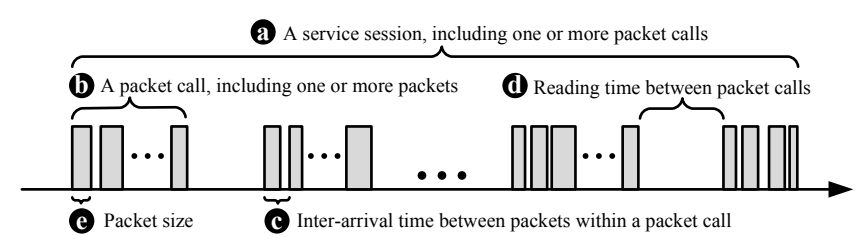

Fig. 4. The characteristic of a service session

destined to the MS. The ETSI model has been widely used to simulate the real packet traffic very well [8], [15].

Figure 4 illustrates the characteristic of a service session defined in the ETSI traffic model. Define the time interval between two consecutive service sessions as "the inter-session idle time", which is modeled as an exponential random variable with mean $1 / \lambda_{i s}$. A service session consists of one or more packet calls (Figure 4 (a)). The number of packet calls in a service session is modeled as a geometric random variable with mean $\mu_{p c}$. In each packet call, there are one or more packets. The number of packets (Figure 4 (b)) in a packet call is modeled as a geometric random variable with mean $\mu_{p}$. The inter-packet arrival time (Figure 4 (c)) within a packet call is modeled as an exponential random variable with mean $1 / \lambda_{p}$. Define the time interval between two consecutive packet calls as "the reading time" (Figure $4(\mathrm{~d})$ ), which is modeled as an exponential random variable with mean $1 / \lambda_{p c}$. The packet size $s_{p}$ (i.e., the number of bytes in a packet; Figure $\left.4(\mathrm{e})\right)$ is modeled as a Pareto random variable with cut-off. A normal Pareto distribution [10] (without cut-off) has two parameters 
$\beta$ and $l$, where $\beta$ describes the "heaviness" of the tail. The probability density function of a normal Pareto distribution is

$$
\begin{aligned}
& f_{P}(x)=\left(\frac{\beta}{l}\right)\left(\frac{l}{x}\right)^{\beta+1}, \\
& \quad \text { and the expected value is } E[P]=\left(\frac{\beta}{\beta-1}\right) l .
\end{aligned}
$$

If $\beta$ is between 1 and 2 , then the variance for the distribution becomes infinity. A upper-bound $m$ is used to set the maximum allowed size of a packet. Following the recommendation in [7], we model the packet size $s_{p}$ as

$$
\text { Packet Size } s_{p}=\min (P, m),
$$

where $P$ is a normal Pareto distributed random variable with $\beta=1.1, l=81.5$ bytes, and $m=66666$ bytes. The mean packet size $E\left[s_{p}\right]$ is calculated in [7] as 480 bytes. The service time $t_{x}$ (with mean $E\left[t_{x}\right]=1 / \lambda_{x}$ ) for a packet is derived from $s_{p}$, and is expressed as

$$
t_{x}=\frac{8 \times \text { Packet Size } s_{p}}{\text { Transmission Bit Rate }} .
$$

Figures 5-7 plot the $P_{i}$ and $E\left[t_{w}\right]$ curves. Following the statistical data in [4], we set $P_{u, b}=1.8, P_{u, i}=0.4, P_{u, s}=$ $0.004, P_{u, l}=0.5, P_{w, o}=0.6$, and $P_{w, f}=0.002$ (all in watts). Other parameter settings are described in the captions of the figures.

Effects of the ratio $\lambda_{f} / \lambda_{o}$. Figures 5-7 show the effects of $\lambda_{f} / \lambda_{o}$ on $P_{i}$ and $E\left[t_{w}\right]$. In the three figures, we observe that in most cases, $P_{i}$ increases as $\lambda_{f} / \lambda_{o}$ increases. The larger $\lambda_{f} / \lambda_{o}$ ratio results in the higher probability that the WLAN connection is available. Thus, less packets are served by the RNC. The WLAN receiver module is likely to be powered on, and the UMTS receiver module is likely to be switched to the sleep mode. When $\lambda_{f} / \lambda_{o}$ increases, $P_{i}$ will be dominated by the power consumption of the WLAN receiver module. As $\lambda_{f} / \lambda_{o}$ increases, it is more likely that the packets are delivered through WLAN with higher transmission rate, and smaller $E\left[t_{w}\right]$ values are observed. In these figures, $E\left[t_{w}\right]$ is a decreasing function of $\lambda_{f} / \lambda_{o}$.

Effects of the mean reading time $1 / \lambda_{p c}$. Figure 5 shows the effects of $1 / \lambda_{p c}$ on $P_{i}$ and $E\left[t_{w}\right]$. Figure 5 (a) shows that when $\lambda_{f} / \lambda_{o}>10^{0}, 1 / \lambda_{p c}$ has minor impact on $P_{i}$. When $\lambda_{f} / \lambda_{o}$ is larger, the MS has a higher probability to stay in a WLAN ON period. In this case, most of the packets are delivered through the WLAN connection, and the UMTS receiver is switched into the sleep mode. Compared with the WLAN power consumption in ON periods, the UMTS power consumption in sleep mode is relatively small and can be ignored. Therefore, changing the packet traffic pattern (via adjusting the reading time) does not have a significant influence on $P_{i}$.

On the other hand, when $\lambda_{f} / \lambda_{o} \leq 10^{0}, P_{i}$ slightly increases when $10^{0} E\left[t_{x}\right] \leq 1 / \lambda_{p c} \leq 10^{3} E\left[t_{x}\right]$, and then slightly decreases when $10^{3} E\left[t_{x}\right] \leq 1 / \lambda_{p c} \leq 10^{5} E\left[t_{x}\right]$. When $\lambda_{f} / \lambda_{o} \leq 10^{0}$, longer WLAN OFF periods are observed, and the packet arrivals have larger chance to be forwarded to the RNC. Therefore, $P_{i}$ performance depends on the behavior of the UMTS receiver. More
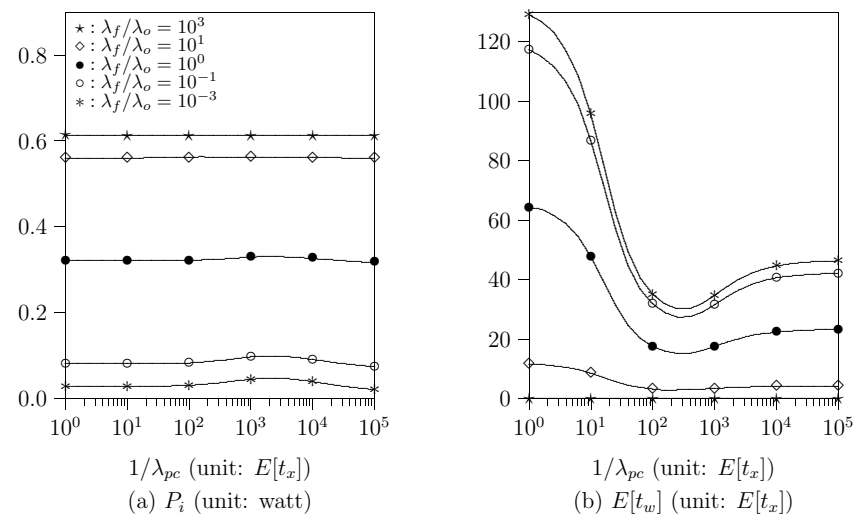

Fig. 5. Effects of $1 / \lambda_{p c}$ and $\lambda_{f} / \lambda_{o}\left(\lambda_{o}=\lambda_{x} / 20000, t_{D}=50 E\left[t_{x}\right]\right.$, $t_{I}=1000 E\left[t_{x}\right], \tau=E\left[t_{x}\right], \lambda_{i s}=\lambda_{x} / 50000, \lambda_{p}=10 \lambda_{x}, \mu_{p c}=5$, and $\left.\mu_{p}=25\right)$

specifically, $P_{i}$ is determined by the ETSI traffic parameters and the power consumption parameters of the UMTS receiver module. Under the considered parameter settings in Figure 5 (a), when $1 / \lambda_{p c} \rightarrow 10^{0} E\left[t_{x}\right], P_{i}$ is dominated by the UMTS sleep mode operation, and the corresponding value is less than 0.4 watts. As $1 / \lambda_{p c}$ increases, the MS experiences longer inactivity periods. Compared with sleep periods, inactivity periods (with power consumption 0.4 watts) are relatively power-consuming. Therefore, $P_{i}$ increases accordingly. However, when $1 / \lambda_{p c}>t_{I}$ (i.e., $1000 E\left[t_{x}\right]$ ), increasing $1 / \lambda_{p c}$ will not further increase the length of inactivity periods. In contrast, longer sleep periods with low power consumption will be observed. Thus, $P_{i}$ decreases as $1 / \lambda_{p c}$ increases.

As shown in Figure 5 (b), when $\lambda_{f} / \lambda_{o}$ is smaller (e.g., $\left.\lambda_{f} / \lambda_{o}<10^{1}\right), E\left[t_{w}\right]$ decreases when $E\left[t_{x}\right] \leq 1 / \lambda_{p c} \leq$ $10^{2} E\left[t_{x}\right]$, and then increases when $10^{3} E\left[t_{x}\right]<1 / \lambda_{p c}<$ $10^{5} E\left[t_{x}\right]$. The smaller $\lambda_{f} / \lambda_{o}$ leads the MS to exercise the UMTS DRX mechanism to receive packets from the RNC. When $1 / \lambda_{p c}<10^{2} E\left[t_{x}\right]$, packets arrive too fast to be served by the RNC, and most of the packets are queued in the RNC buffer. For this reason, the mean packet waiting time $E\left[t_{w}\right]$ increases as $1 / \lambda_{p c}$ decreases. When $1 / \lambda_{p c} \geq 10^{3} E\left[t_{x}\right]$, the MS enters the power saving mode more frequently, and the $E\left[t_{w}\right]$ increases gradually as $1 / \lambda_{p c}$ increases. We note that the above effect is less obvious in higher $\lambda_{f} / \lambda_{o}$ cases (e.g., $\lambda_{f} / \lambda_{o}=10^{3}$ ). This is because higher $\lambda_{f} / \lambda_{o}$ ratio results in that packets are mainly served by the WLAN, and these packets can be delivered immediately.

Effects of the length of a DRX cycle $t_{D}$. Figure 6 (a) shows the intuitive result that $P_{i}$ is a decreasing function of the length of a DRX cycle $t_{D}$. Power consumption in the power saving mode is influenced by $\tau / t_{D}$. In this figure, we set $\tau$ as a fixed value. As $t_{D}$ increases, the proportion of the listening period in a DRX cycle decreases, and therefore $P_{i}$ decreases accordingly. We observe that when $t_{D}>10^{2} E\left[t_{x}\right]$, the listening period in a DRX cycle is always within $1 \%$. Consequently, increasing $t_{D}$ can not further improve the $P_{i}$ performance. 

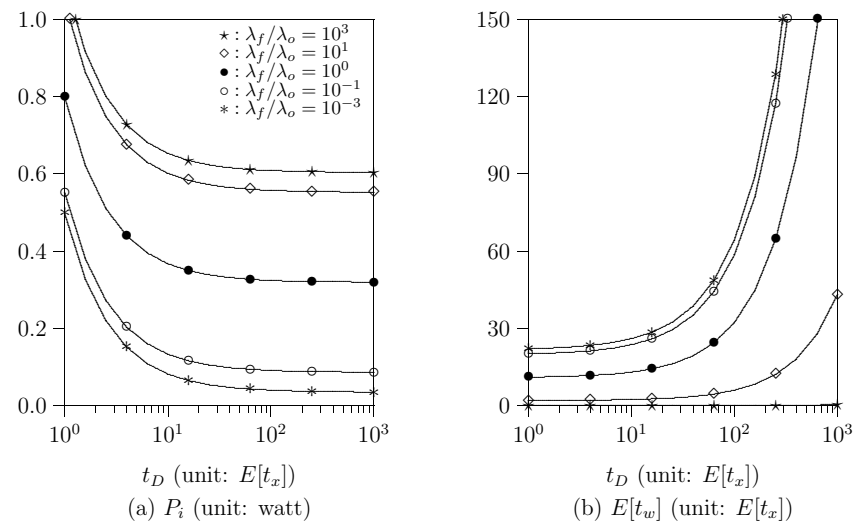

Fig. 6. Effects of $t_{D}$ and $\lambda_{f} / \lambda_{o}\left(\lambda_{o}=\lambda_{x} / 20000, t_{I}=1000 E\left[t_{x}\right]\right.$, $\tau=E\left[t_{x}\right], \lambda_{i s}=\lambda_{x} / 50000, \lambda_{p c}=\lambda_{x} / 5000, \lambda_{p}=10 \lambda_{x}, \mu_{p c}=5$, and $\left.\mu_{p}=25\right)$

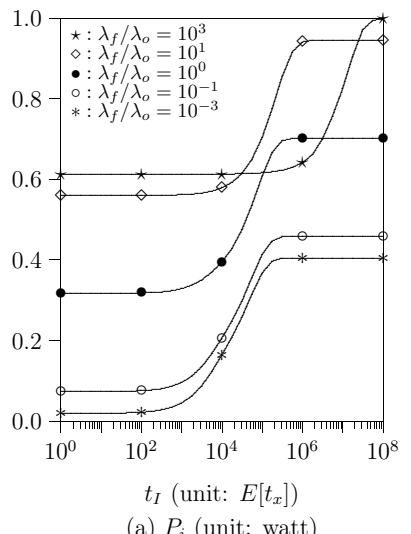

(a) $P_{i}$ (unit: watt)

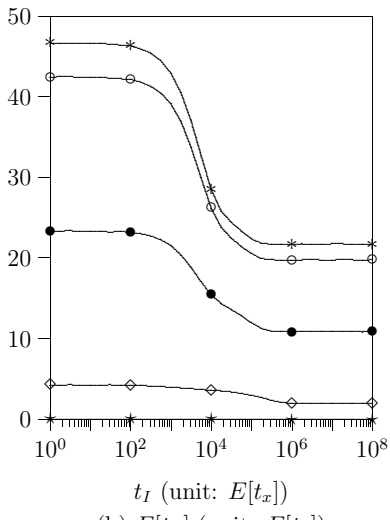

(b) $E\left[t_{w}\right]$ (unit: $\left.E\left[t_{x}\right]\right)$
Fig. 7. Effects of $t_{I}$ and $\lambda_{f} / \lambda_{o}\left(\lambda_{o}=\lambda_{x} / 20000, t_{D}=50 E\left[t_{x}\right], \tau=\right.$ $E\left[t_{x}\right], \lambda_{i s}=\lambda_{x} / 50000, \lambda_{p c}=\lambda_{x} / 5000, \lambda_{p}=10 \lambda_{x}, \mu_{p c}=5$, and $\left.\mu_{p}=25\right)$

Figure 6 (b) shows that $E\left[t_{w}\right]$ is an increasing function of $t_{D}$. When $t_{D}$ is larger (e.g., $t_{D}>10 E\left[t_{x}\right]$ ), the probability that a packet arrives during a sleep period is higher. The $E\left[t_{w}\right]$ significantly increases as $t_{D}$ increases. With a small $t_{D}$ value (e.g., $t_{D}<10 E\left[t_{x}\right]$ ), the MS is most likely to be in a listening period even if the packet arrives during a DRX cycle (i.e., the MS wakes up to listen to the paging channel), and $E\left[t_{w}\right]$ is dominated by the waiting time in the RNC buffer. In this case, decreasing $t_{D}$ rarely improves the $E\left[t_{w}\right]$ performance. From the above observations, we suggest that $t_{D}$ should be selected in the range $\left[10 E\left[t_{x}\right], 100 E\left[t_{x}\right]\right]$.

\section{Effects of the threshold $t_{I}$ of the RNC inactivity timer.}

With smaller $t_{I}$, the UMTS receiver module enters the sleep mode soon after each busy period, which lowers $P_{i}$ and increases $E\left[t_{w}\right]$. Figure 7 (a) shows a general phenomenon that $P_{i}$ remains stable when $t_{I}$ is smaller than a small threshold $\epsilon_{s}$, then increases rapidly when $\epsilon_{s}<t_{I}<\epsilon_{l}$ (where $\epsilon_{l}$ is another threshold larger than $\epsilon_{s}$ ), and then turns to be stable when $t_{I}>\epsilon_{l}$. For example, for the " $*$ " curve (i.e., $\lambda_{f} / \lambda_{o}=10^{-3}$ ), $\epsilon_{s} \simeq 10^{2} E\left[t_{x}\right]$ and $\epsilon_{l} \simeq 10^{5} E\left[t_{x}\right]$. When $t_{I}<\epsilon_{s}$, packets are not likely to arrive before the RNC timer expires, and the MS can enter the sleep mode to reduce the power consumption. With increasing of $t_{I}$, this effect becomes minor, and we observe that $P_{i}$ increases rapidly. When $t_{I}>\epsilon_{l}$, the MS almost always stays in busy or inactivity periods. Hence, no power saving is possible, and stable and larger $P_{i}$ values are observed. We also note that the values of $\epsilon_{s}$ and $\epsilon_{l}$ depend on the setting of $\lambda_{f} / \lambda_{o}$. As $\lambda_{f} / \lambda_{o}$ increases, $\epsilon_{s}$ and $\epsilon_{l}$ increase.

As shown in Figure 7 (b), for $E\left[t_{w}\right]$, we observe the opposite phenomenon. That is, $E\left[t_{w}\right]$ remains stable when $t_{I}$ is smaller than a small threshold $\epsilon_{s}$, then decreases rapidly when $\epsilon_{s}<t_{I}<\epsilon_{l}$ (where $\epsilon_{l}$ is another threshold larger than $\epsilon_{s}$ ), and then turns to be stable when $t_{I}>\epsilon_{l}$. The reason is given as follows. A larger $t_{I}$ value implies a higher probability that the MS stays in an inactivity period when packets arrive. In this case, the packets have better chance to be served immediately, and lower $E\left[t_{w}\right]$ is expected.

\section{Conclusions}

This paper proposed an analytical model and conducted simulation experiments to investigate the power consumption of a dual-mode MS in terms of the power consumption indicator $P_{i}$ and the mean packet waiting time $E\left[t_{w}\right]$. Our study indicated the following. The $\lambda_{f} / \lambda_{o}$ ratio has conflicting impacts on $P_{i, w}$ and $P_{i, u}$. More specifically, as $\lambda_{f} / \lambda_{o}$ increases, $P_{i, w}$ increases while $P_{i, u}$ decreases. We observed reverse effects of $\lambda_{f} / \lambda_{o}$ on $E\left[t_{w}\right]$, and $E\left[t_{w}\right]$ becomes more sensitive for a smaller $\lambda_{f} / \lambda_{o}$ than a larger $\lambda_{f} / \lambda_{o}$. Under the parameter settings recommended in the ETSI traffic model, $P_{i}$ increases and then decreases as the mean reading time increases, and $t_{D}$ should be chosen from the range $\left[10 E\left[t_{x}\right], 100 E\left[t_{x}\right]\right]$. We also found that in some $t_{I}$ settings, a higher $\lambda_{f} / \lambda_{o}$ consumes less power than a lower $\lambda_{f} / \lambda_{o}$. We gave an interpretation for this phenomenon.

\section{ACKNOWLEDGMENT}

We would like to thank the anonymous reviewers. Their valuable comments have significantly enhanced the quality of this paper.

\section{APPENDIX}

This section describes the discrete event-driven simulation model that simulates the power consumption behavior of an MS in the GAN and UMTS interworking environment. We define six types of events listed as follows:

- The ARRIVAL event represents a packet arrival to the SGSN.

- The DEPARTURE event implies that a packet has been successfully transmitted to the MS.

- The WLAN_ON event represents that WLAN connectivity enters an ON period from an OFF period, i.e., the WLAN connection becomes available.

- The WLAN_OFF event represents that WLAN connectivity enters an OFF period from an ON period, i.e., the WLAN connection becomes unavailable.

- The SLEEP event represents that an inactivity period of the MS ends, and the MS enters a sleep period. 
- The WAKEUP event represents that the MS wakes up from a sleep period.

A timestamp is maintained in each event to indicate when the event occurs. Events are inserted into an event list and are deleted/processed from the list in a non-decreasing timestamp order. During the execution of simulation, a simulation clock $t_{s}$ is maintained to indicate the progress of the simulation. The following variables are used in the simulation model:

- $t_{a}$ stores the inter-packet arrival time.

- $t_{x}$ stores the packet service time.

- $t_{o}$ stores the length of a WLAN ON period.

- $t_{f}$ stores the length of a WLAN OFF period.

The following counters are used in our simulation model to calculate the output measures (to be elaborated on later):

- $N_{a}$ counts the total number of ARRIVAL events.

- $N_{w}$ counts the total number of WAKEUP events.

- $T_{b}$ calculates the total time when the MS is in the UMTS busy periods.

- $T_{i}$ calculates the total time when the MS is in the UMTS inactivity periods.

- $T_{s}$ calculates the total time when the MS is in the UMTS sleep periods.

- $T_{o}$ calculates the total time when the MS is in the WLAN ON periods.

- $T_{f}$ calculates the total time when the MS is in the WLAN OFF periods.

- $T_{w}$ calculates the total waiting time for queued packets.

We repeat the simulation runs until $N_{a}$ exceeds the constant MAXARRIVAL, which is a fixed and sufficiently large positive number, to ensure the stability of the simulation results. Two output measures are investigated in our model, including the power consumption indicator $P_{i}$ and the mean packet waiting time $E\left[t_{w}\right]$, which are obtained as follows (equation 23): and

$$
E\left[t_{w}\right]=\frac{T_{w}}{N_{a}},
$$

where $P_{u, b}, P_{u, i}, P_{u, s}, P_{u, l}, P_{w, o}, P_{w, f}$ and $\tau$ are defined in Section III.

Figure 8 illustrates the flowchart of our simulation model. The variable UmtsState stores the state of the MS's UMTS receiver, whose value can be busy, inactivity or sleep. The variable Wlanstate stores the state of the MS's WLAN receiver, whose value can be on or off. The two variables $t_{p, u}$ and $t_{p, w}$ store the times when the last changes of Umts State and WlanState occur, respectively. We maintain a FIFO list denoted as RNC_buffer to buffer the ARRIVAL events that can not be served immediately. The execution of the simulation model is described as follows.

Step 1 sets up the input parameters (including $\lambda_{o}, \lambda_{f}$, $t_{I}, t_{D}$, etc.). In addition, the counters (e.g., $N_{a}, N_{w}, T_{b}$, $T_{s}, T_{f}, t_{s}$, and $\left.t_{p, w}\right)$ are set to zero, and the event list is set to NULL. Step 2 sets Umts State to inactivity. Step 3 generates a WLAN_ON event and an ARRIVAL event. The timestamp of the WLAN_ON event is set to 0 . We generate a $t_{a}$ value following the exponential distribution with rate $\lambda_{a}$ or following the ETSI traffic model. The timestamp of the ARRIVAL event is set to $t_{a}$. The WLAN_ON and ARRIVAL

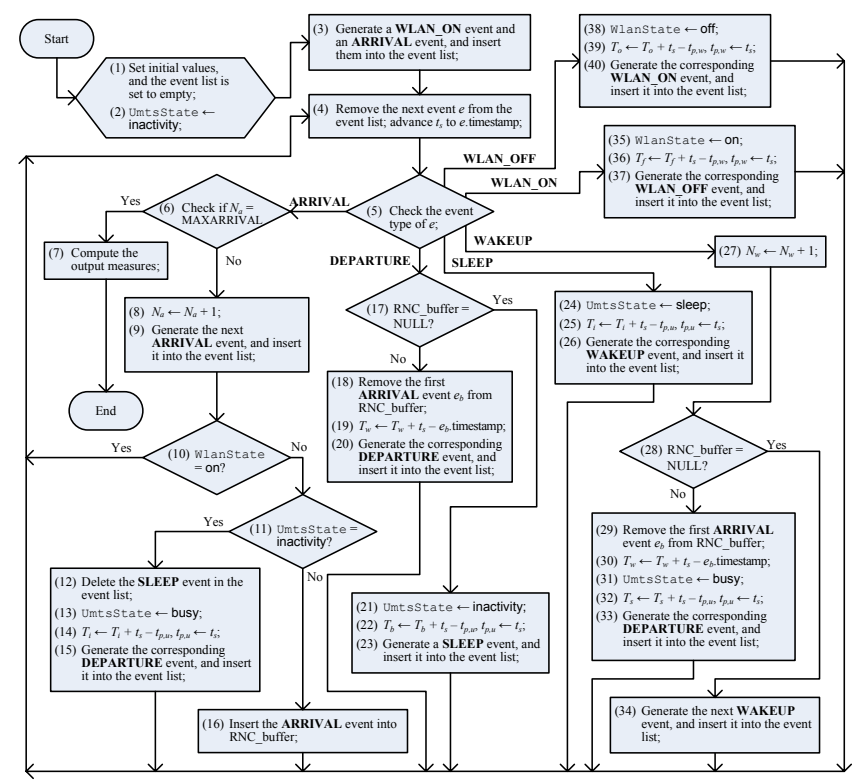

Fig. 8. Flowchart of the simulation model

events are inserted into the event list. Step 4 removes the next event $e$ from the event list, and set the value of $t_{s}$ to $e$.timestamp. Step 5 checks the type of event $e$.

If event $e$ is an ARRIVAL event at Step 5, Step 6 checks whether $N_{a}$ is equal to MAXARRIVAL. If so, Step 7 computes the output measures using (23) and (24), and the simulation terminates. Otherwise (i.e., $N_{a}<$ MAXARRIVAL), Step 8 increases $N_{a}$ by one. Step 9 generates the next ARRIVAL event, whose timestamp is set to $t_{s}+t_{a}$ (where $t_{a}$ is randomly generated following the exponential distribution with rate $\lambda_{a}$ or is generated following the ETSI traffic model). Then this new ARRIVAL event is inserted into the event list. Step 10 checks whether WlanState is equal to on. If so, the simulation goes back to Step 4 . This is because the WLAN connection supports high data transmission rate, and we assume that the service time and waiting time of the ARRIVAL event $e$ in GAN can be ignored. On the other hand, if Wlanstate is equal to off, Step 11 checks whether UmtsState is equal to inactivity. If so (i.e., the MS is in an inactivity period), due to the packet arrival, the MS enters a new busy period. Step 12 removes the SLEEP event (corresponding to this inactivity period) from the event list. Step 13 sets Umts State to busy. Step 14 sets $T_{i} \leftarrow T_{i}+t_{s}-t_{p, u}$ and $t_{p, u} \leftarrow t_{s}$. Step 15 generates a DEPARTURE event (corresponding to this ARRIVAL event) with timestamp $t_{s}+t_{x}$ (where $t_{x}$ is randomly generated following the general distribution with mean $1 / \lambda_{x}$ and variance $V_{x}$ or is generated following the ETSI traffic model), and then inserts it into the event list. If UmtsState $\neq$ inactivity at Step 11 (i.e., UmtsState = busy or sleep), the packet arrival can not be served. Step 16 inserts the ARRIVAL event into the RNC_buffer list.

If event $e$ is a DEPARTURE event at Step 5, Step 17 checks whether there is any ARRIVAL event in the RNC_buffer list. If so, Steps 18-20 are executed to select a buffered packet arrival for transmission. Step 18 removes the first ARRIVAL event (denoted as $e_{b}$ ) from the RNC_buffer list. Step 19 sets 


$$
P_{i}=\frac{T_{b} P_{u, b}+T_{i} P_{u, i}+\left(T_{s}-N_{w} \tau\right) P_{u, s}+N_{w} \tau P_{u, l}+T_{o} P_{w, o}+T_{f} P_{w, f}}{t_{s}}
$$

$T_{w} \leftarrow T_{w}+t_{s}-e_{b}$.timestamp. Step 20 generates a DEPARTURE event (corresponding to the ARRIVAL event $e_{b}$ ) with timestamp $t_{s}+t_{x}$ (where $t_{x}$ is randomly generated following the general distribution with mean $1 / \lambda_{x}$ and variance $V_{x}$ or is generated following the ETSI traffic model), and then inserts it into the event list. The simulation goes back to Step 4 . If the RNC_buffer list is NULL at Step 17, Step 21 sets Umt s State to inactivity. At Step $22, T_{b}$ is set to $T_{b}+t_{s}-t_{p, u}$ and $t_{p, u}$ is set to $t_{s}$. Step 23 generates a SLEEP event with timestamp $t_{s}+t_{I}$, and then inserts it into the event list. The simulation returns to Step 4.

If event $e$ is a SLEEP event at Step 5, Umt sState is set to sleep at Step 24. We update $T_{i}$ and $t_{p, u}$ as $T_{i} \leftarrow T_{i}+t_{s}-t_{p, u}$ and $t_{p, u} \leftarrow t_{s}$ at Step 25. Step 26 generates a WAKEUP event with timestamp $t_{s}+t_{D}$, and then inserts it into the event list. The simulation goes back to Step 4 .

If event $e$ is a WAKEUP event at Step 5, Step 27 increases $N_{w}$ by one. Step 28 checks whether the RNC_buffer list is NULL. If not (i.e., there are buffered packet arrivals), Step 29 removes the first ARRIVAL event $e_{b}$ from the RNC_buffer list to process. Step 30 sets $T_{w} \leftarrow T_{w}+t_{s}-e_{b}$.timestamp. The UmtsState is set to busy at Step 31. Step 32 updates $T_{s}$ and $t_{p, u}$ as $T_{s} \leftarrow T_{s}+t_{s}-t_{p, u}$ and $t_{p, u} \leftarrow t_{s}$. Step 33 generates a DEPARTURE event corresponding to the ARRIVAL event $e_{b}$ with timestamp $t_{s}+t_{x}$ (where $t_{x}$ is randomly generated following the general distribution with mean $1 / \lambda_{x}$ and variance $V_{x}$ or is generated following the ETSI traffic model), and then inserts it into the event list. If the RNC_buffer list is NULL at Step 28, Step 34 generates the next WAKEUP event with timestamp $t_{s}+t_{D}$, and then inserts it into the event list. The simulation returns to Step 4.

If event $e$ is a WLAN_ON event at Step 5, Steps 3537 are executed to activate a WLAN ON period. Step 35 sets WlanState to on. Step 36 updates $T_{f}$ and $t_{p, w}$ as $T_{f} \leftarrow T_{f}+t_{s}-t_{p, w}$ and $t_{p, w} \leftarrow t_{s}$, respectively. Step 37 generates a WLAN_OFF event (corresponding to this WLAN ON period) with timestamp $t_{s}+t_{o}$ (where $t_{o}$ is randomly generated following the exponential distribution with rate $\lambda_{o}$ ), and then inserts it into the event list. The simulation goes back to Step 4.

If event $e$ is a WLAN_OFF event at Step 5, Step 38 sets WlanState to off. Step 39 updates $T_{o}$ and $t_{p, w}$ as $T_{o} \leftarrow T_{o}+t_{s}-t_{p, w}$ and $t_{p, w} \leftarrow t_{s}$, respectively. Step 40 generates a WLAN_ON event (corresponding to this WLAN OFF period) with timestamp $t_{s}+t_{f}$ (where $t_{f}$ is randomly generated following the exponential distribution with rate $\lambda_{f}$ ), and then inserts the generated event into the event list. The simulation returns to Step 4.

\section{REFERENCES}

[1] "3rd generation partnership project; technical specification group GSM/EDGE radio access network. Generic access to the A/Gb interface; stage 2," Technical Specification 3GPP TS 43.318 version 6.6.0, 3GPP, April 2006.
[2] "3rd generation partnership project; technical specification group radio access network, RRC protocol specification for release 1999," Technical Specification, 3GPP TS 25.331 version 3.5.0, 3GPP, December 2000.

[3] "Bluetooth Special Interest Group, Bluetooth Core Specification v2.0," Technical Standard, Bluetooth SIG, Nov. 2004.

[4] Z.-H. Chen and J.-C. Lin, "Technical Slides: WCDMA current power consumption," Inventec Appliances Corp., Dec. 2006.

[5] J. N. Daigle, Queueing Theory for Telecommunications. AddisonWesley, 1992.

[6] A. Doufexi, E. Tameh, A. Nix, S. Armour, and A. Molina, "Hotspot wireless LANs to enhance the performance of $3 \mathrm{G}$ and beyond cellular networks," IEEE Commun. Mag., vol. 41, no. 7, pp. 58-65, July 2003.

[7] European Telecommunications Standards Institute, "Universal mobile telecommunications system (UMTS); selection procedures for the choice of radio transmission technologies of the UMTS," Technical Report: TR 101112 UMTS 30.03, version 3.2.0, ETSI, Apr. 1998.

[8] S. Hämäläinen, H. Holma, and K. Sipilä, "Advanced WCDMA radio network simulator," in Proc. PIMRC, pp. 509-604, Sept. 1999.

[9] "IEEE, wireless medium access control (MAC) and physical layer (PHY) specifications," Technical Standard, IEEE Standard 802.11, IEEE, Apr. 1997.

[10] N. L. Johnson, Continuous Univariate Distributions, volume 1. John Wiley\& Sons, 1970.

[11] A. M. Law, and W. D. Kelton, Simulation Modeling and Analysis. McGraw-Hill Higher Education, 3rd edition, 2000.

[12] C.-C. Lee, J.-H. Yeh, and J.-C. Chen, "Impact of inactivity timer on energy consumption in WCDMA and cdma2000," in Proc. Wireless Telecommun. Symposium, May 2004.

[13] P. Lin, Y.-B. Lin, and I. Chlamtac, "Modeling frame synchronization for UMTS high-speed downlink packet access," IEEE Trans. Veh. Technol., vol. 52, no. 1, pp. 132-141, Jan. 2003.

[14] Y.-B. Lin and Y.-M. Chuang, "Modeling the sleep mode for cellular digital packet data," IEEE Commun. Lett., vol. 3, no. 3, pp. 63-65, Mar. 1999.

[15] H. N. Nguyen and I. Sasase, "Downlink queuing model and packet scheduling for providing lossless handoff and QoS in 4G mobile networks," IEEE Trans. Mobile Comput., vol. 5, no. 5, pp. 452-462, May 2006.

[16] S. M. Ross, Stochastic Processes. New York: John Wiley, 2nd edition, 1996.

[17] E. Shih, P. Bahl, and M. J. Sinclair, "Wake on wireless: an event driven energy saving strategy for battery operated devices." in Proc. MobiCom, Sept. 2002.

[18] "UMA Consortium, Unlicensed Mobile Access (UMA) Architecture (Stage 2)," R1.0.0, Technical Specification, Sept. 2004.

[19] S.-R. Yang and Y.-B. Lin, "Modeling UMTS discontinuous reception mechanism," IEEE Trans. Wireless Commun., vol. 4, no. 1, pp. 312319, Jan. 2005.

[20] R. Zheng, J. C. Hou, and L. Sha, "Performance analysis of power management policies in wireless networks," IEEE Trans. Wireless Commun., vol. 5 , no. 6 , pp. 1351-1361, June 2006.

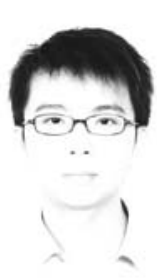

Shun-Ren Yang (M'08) received the BSCSIE, MSCSIE, and Ph.D. degrees from National Chiao Tung University, Hsinchu, Taiwan, R.O.C., in 1998, 1999, and 2004, respectively. From April 1, 2004 to July 31, 2004, he was appointed as a Research Assistant in the Department of Information Engineering, the Chinese University of Hong Kong. Since August 2004, he has been with the Department of Computer Science and Institute of Communications Engineering, National Tsing Hua University, Taiwan, where he is an Assistant Professor. His current research interests include design and analysis of personal communications services networks, computer telephony integration, mobile computing, and performance modeling. 


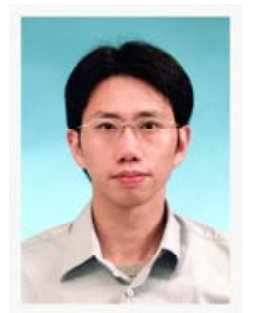

Phone Lin (M'02-SM'06) received his BSCSIE degree and Ph.D. degree from National Chiao Tung University, Taiwan, R.O.C. in 1996 and 2001, respectively. From August 2001 to July 2004 and from August 2004 to July 2008, he was an Assistant Professor and Associate Professor in Department of Computer Science and Information Engineering (CSIE), National Taiwan University, R.O.C. Since August 2008, he has been a Professor in Department of CSIE and in Graduate Institute of Networking and Multimedia, National Taiwan University, R.O.C. His current research interests include personal communications services, wireless Internet, and performance modeling. Dr. Lin has published more than twenty international SCI journal papers (most of which are IEEE Transactions and ACM papers). Dr. Lin is an Associate Editor for IEEE TRANSACTIONS ON Vehicular TEChNOLOGY, a Guest Editor for IEEE Wireless COMMUNICATIONS special issue on Mobility and Resource Management, and a Guest Editor for ACM/SPRINGER MONET special issue on Wireless Broad Access. $\mathrm{He}$ is also an Associate Editorial Member for the WCMC JOURNAL. Dr. Lin has received many research awards. He was elected as the Best Young Researcher, the 3rd IEEE ComSoc Asia-Pacific Young Researcher Award, 2007. He was a recipient of Research Award for Young Researchers from
Pan Wen-Yuan Foundation in Taiwan in 2004, a recipient of K. T. Li Young Researcher Award honored by ACM Taipei Chapter in 2004, a recipient of Wu Ta You Memorial Award of National Science Council (NSC) in Taiwan in 2005, a recipient of Fu Suu-Nien Award of NTU in 2005 for his research achievements, and a recipient of 2006 Young Electrical Engineering Award, the Chinese Institute of Electrical Engineering. Dr. Lin is listed in Who's Who in Science and Engineering(R) in 2006. Dr. Lin is a Senior Member, IEEE. P. Lin's e-mail and website address are plin@csie.ntu.edu.tw and http://www.csie.ntu.edu.tw/ plin/, respectively.

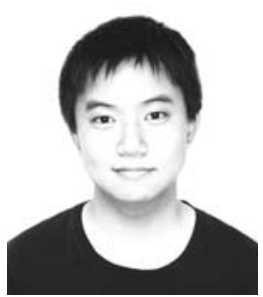

Pei-Tang Huang received the BSCSIE degree from Tamkang University, Taipei County, Taiwan, R.O.C., in 2005, and the MSCS degree from National Taiwan University, Taipei, Taiwan, R.O.C., in 2007. In 2007, he joined the Telecommunication Laboratories, Chunghwa Telecom Company Ltd., Taiwan, R.O.C. His research interests include the design and analysis of the personal communications services network, and the development of telecommunication operation support systems. 